\title{
Application of quasi-equilibrated thermodesorption of hexane and cyclohexane for characterization of porosity of zeolites and ordered mesoporous silicas
}

\author{
Wacław Makowski • Maria Mańko • \\ Anna Dudek · Kinga Mlekodaj
}

Received: 30 November 2012/ Accepted: 17 January 2013/Published online: 25 January 2013

(C) The Author(s) 2013. This article is published with open access at Springerlink.com

\begin{abstract}
Quasi equilibrated temperature programmed desorption and adsorption (QE-TPDA) of hexane and cyclohexane was applied for characterization of zeolites 5A, ZSM-5, 13X, Y, NaMOR and ordered mesoporous silicas MCM-41, MCM-41/TMB, SBA-15 and HMS. Similar QE-TPDA profiles of hexane and cyclohexane with a single desorption maximum were observed for the wide pore zeolites. No adsorption of cyclohexane for zeolite $5 \mathrm{~A}$ and a single desorption maximum for ZSM-5 were found, while two-step desorption profiles of hexane were observed for these zeolites. Similar values of the adsorption enthalpy and entropy of hexane and cyclohexane were obtained by fitting the Langmuir model functions for the zeolites $\mathrm{X}$ and Y. For NaMOR and ZSM-5 larger differences in these parameters were found. A single desorption peak found at low temperatures in the QE-TPDA profiles of hexane and cyclohexane for the studied silicas was attributed to the multilayered adsorption on their mesopore surface. The adsorption isobars calculated from the thermodesorption profiles were fitted with the BET function. This way values of the specific surface area and the adsorption heat were calculated. Additionally values of the initial heat of adsorption were found by fitting the Henry's law to the high-temperature sections of the linearized isobars. The largest deviations from the BET and Henry functions and the largest values of the adsorption heats found for SBA-15 indicated the greatest heterogeneity of the adsorption sites on its surface.
\end{abstract}

Keywords Hexane - Cyclohexane - Adsorption heat . Zeolite $\cdot$ Mesoporous silica $\cdot$ Surface heterogeneity

W. Makowski $(\bowtie) \cdot$ M. Mańko · A. Dudek · K. Mlekodaj Faculty of Chemistry, Jagiellonian University, Kraków, Poland e-mail: makowski@chemia.uj.edu.pl

\section{Introduction}

Porosity of solids plays an important role in many technological applications, mainly in separation and heterogeneous catalysis. Main tools for characterization of porosity are based on measurements of physisorption isotherms. Low temperature $\mathrm{N}_{2}$ adsorption is standard method for determination of the specific surface area, micro- and mesopore volume, and the pore size distribution. Extensive reference data and numerous theoretical models and computational methods are available for various porous materials, e.g. activated carbons and micelle templated mesoporous silicas. However, for low silica zeolites, $\mathrm{N}_{2}$ adsorption is not appropriate for porosity characterization, as quadrupole interactions of $\mathrm{N}_{2}$ molecules with the extraframework cations and the framework $\mathrm{Al}$ atoms result in prolonged equilibration times and pore filling patterns that do not correlate with the micropore sizes (Thommes 2010). Adsorption of Ar at $87 \mathrm{~K}$ gives much better results, but it is still not widely used.

Interactions of $n$-alkanes with molecular sieves were extensively studied in recent years, because of their importance in hydrocarbon processing technologies, therefore numerous reference data are available in the literature. Hexane and cyclohexane are good probe molecules for studying porosity of zeolites and mesoporous silicas due to their low chemical reactivity and appropriate size and shape of the molecules (Traa et al. 2007). Adsorption of hexane was recommended as a method for determination of the micropore volume of zeolites (Ruthven 2001). The ratio of saturation adsorption capacity of cyclohexane to that of hexane was introduced as the shape selectivity index allowing discrimination between the 8, 10 and $12 \mathrm{MR}$ pores in zeolites (Otake 1993). Size of the micropores in zeolites may be estimated from the correlation between the 
adsorption enthalpy and entropy observed for a series of $n$ alkanes (Eder and Lercher 1997). A quantitative relation between the adsorption enthalpy of $n$-alkanes and the zeolites micropore size was found for different pore geometries (Roque-Malherbe and Diaz-Castro 2008).

Quasi equilibrated temperature programmed desorption and adsorption (QE-TPDA) is a new experimental technique developed as a method for characterization of porous materials (Makowski 2007). QE-TPDA measurements are performed by controlled cyclic heating and cooling the studied sample in a flow system equipped with a TCD detector, using He with small admixture of an adsorptive as a carrier gas. Quasi-equilibrium control of such measurements, i.e. the fact that despite simultaneous changes of temperature and adsorptive partial pressure the adsorption degree depends only on the adsorption-desorption equilibrium, is supported both by the statistical rate theory (Pańczyk et al. 2006) and experimental proofs (Makowski 2007).

QE-TPDA of $n$-alkanes has been found to be a good method for characterization of zeolites (Makowski and Ogorzałek 2007), mesoporous silicas (Makowski et al. 2009; Mańko et al. 2010) and their carbons replicas (Makowski et al. 2010). It allows detection of the micropores and mesopores, determination of their volume and the mesopore size distribution as well as the adsorption enthalpy and entropy. The aim of this work was a comparison of the QE-TPDA profiles of cyclohexane and hexane for selected zeolites and micelle-templated silicas, differing in geometry of the pores. Discrimination of the studied materials based on the obtained experimental parameters characterizing their porosity and surface chemistry, in relation to the size and shape of the probe molecules, was attempted.

\section{Experimental}

The studied zeolites were commercial ZSM-5 ( $\mathrm{Si} / \mathrm{Al}=$ 140, CBV 28014 Zeolyst), 13X (NaX, Si/Al = 1.25, Supelco, for GC), Y (HY, Si/Al = 15, CBV 720 Zeolyst), $5 \mathrm{~A}(\mathrm{CaA}, \mathrm{Si} / \mathrm{Al}=1.0)$, obtained by ion exchange from $\mathrm{NaA}$ (Siliporite NK-10 Arkema) and NaMOR $(\mathrm{Si} / \mathrm{Al}=$ 10), obtained by ion exchange from $\mathrm{NH}_{4} \mathrm{MOR}$ (CBV 21A Zeolyst). The zeolites $5 \mathrm{~A}$ and NaMOR were prepared by stirring $0.5 \mathrm{~g}$ of a parent zeolite in $50 \mathrm{~mL}$ of $1 \mathrm{M}$ nitrate solution for $20 \mathrm{~h}$ at $60{ }^{\circ} \mathrm{C}$. The ion-exchanged zeolites were filtered, washed with deionized water and dried overnight.

The studied ordered mesoporous silicas were synthesized in presence of long-chain quaternary ammonium cations (without or with addition of 1.3.5-trimethylbenzene-MCM-41 and MCM-41/TMB, respectively), hexadecyltrimethylamine (HMS) and triblock copolymer poly(ethylene oxide) - poly(propylene oxide) (SBA-15) as surfactants. The detailed synthesis procedures and porosity characteristics were published earlier (Majda et al. 2012).

The measurements of quasi-equilibrated temperature programmed desorption and adsorption of hexane and cyclohexane (analytical pure, $\mathrm{POCh}$ ) were performed using a TPD setup equipped with a chromatographic thermal conductivity detector (Micro Volume TCD, Valco), which was described in more details earlier (Makowski 2007; Makowski and Ogorzałek 2007; Mańko et al. 2010). Small samples of the studied materials (ca $10 \mathrm{mg}$ of zeolite, ca $4 \mathrm{mg}$ of silica) were used. Before each experiment, the sample was activated by heating in a flow of pure $\mathrm{He}$ at $10{ }^{\circ} \mathrm{C} / \mathrm{min}$ to $500{ }^{\circ} \mathrm{C}$. The QE-TPDA experiments were performed by heating the sample with the preadsorbed hexane or cyclohexane in a flow of He/adsorptive mixture $\left(6.5 \mathrm{~cm}^{3} / \mathrm{min}\right)$ according to a temperature program consisting of several heating and cooling ramps at $10{ }^{\circ} \mathrm{C} / \mathrm{min}$ to $500{ }^{\circ} \mathrm{C}$ for zeolites and to $250^{\circ} \mathrm{C}$ for mesoporous silicas.

The pore volumes were determined from the thermodesorption profiles by integrating the desorption maxima and referring the obtained area to the appropriate calibration data, assuming density of the adsorptive equal to that of the liquid.

\section{Results and discussion}

\subsection{Zeolites}

The QE-TPDA profiles of hexane and cyclohexane obtained for the studied zeolites are compared in Figs. 1 and 2. For the narrow and medium pore zeolites large differences in thermodesorption of hexane and cyclohexane were found (Fig. 1). While QE-TPDA profiles of hexane showed considerable intensity for both ZSM-5 and $5 \mathrm{~A}$, in the profiles of cyclohexane no desorption was observed for $5 \mathrm{~A}$ and only a high temperature peak for ZSM-5. The two step profiles of hexane observed for 5A and ZSM-5 zeolites are in agreement with the earlier findings (Sivasankar and Vasudevan 2005) and may be explained in terms of the "commensurate freezing" effect, i.e. an ordering of the molecules adsorbed in the three dimensional micropore system, resulting from matching of its periodicity with the length of the molecule (Smit and Maesen 1995).

For the wide pore zeolites the profiles observed for both hydrocarbons are quite similar, only in the case of NaMOR intensity of the thermodesorption peaks was smaller for cyclohexane. For this zeolite also some effects due to catalytic reaction hydrocarbons were observed at high 
temperatures-increase of the detector signal indicating cracking, accompanied by coking leading to decrease of the adsorption capacity. These effects were not observed in the additional QE-TPDA measurements were performed in the temperature range $25-400{ }^{\circ} \mathrm{C}$ (not shown here).

The thermodesorption data obtained for the studied zeolites were interpreted quantitatively by fitting with functions based on the Langmuir adsorption model, according to the procedure published earlier (Makowski and Ogorzałek 2007). The QE-TPDA profiles were integrated and normalized to one. Adsorption isobars were calculated by averaging of the integral desorption and adsorption profiles. In the case of NaMOR the QE-TPDA profiles recorded up to $400{ }^{\circ} \mathrm{C}$, not affected by catalytic reactions, were used. Temperature derivatives of the experimental isobars exhibiting a single minimum were fitted with the temperature derivative of the Langmuir adsorption function:

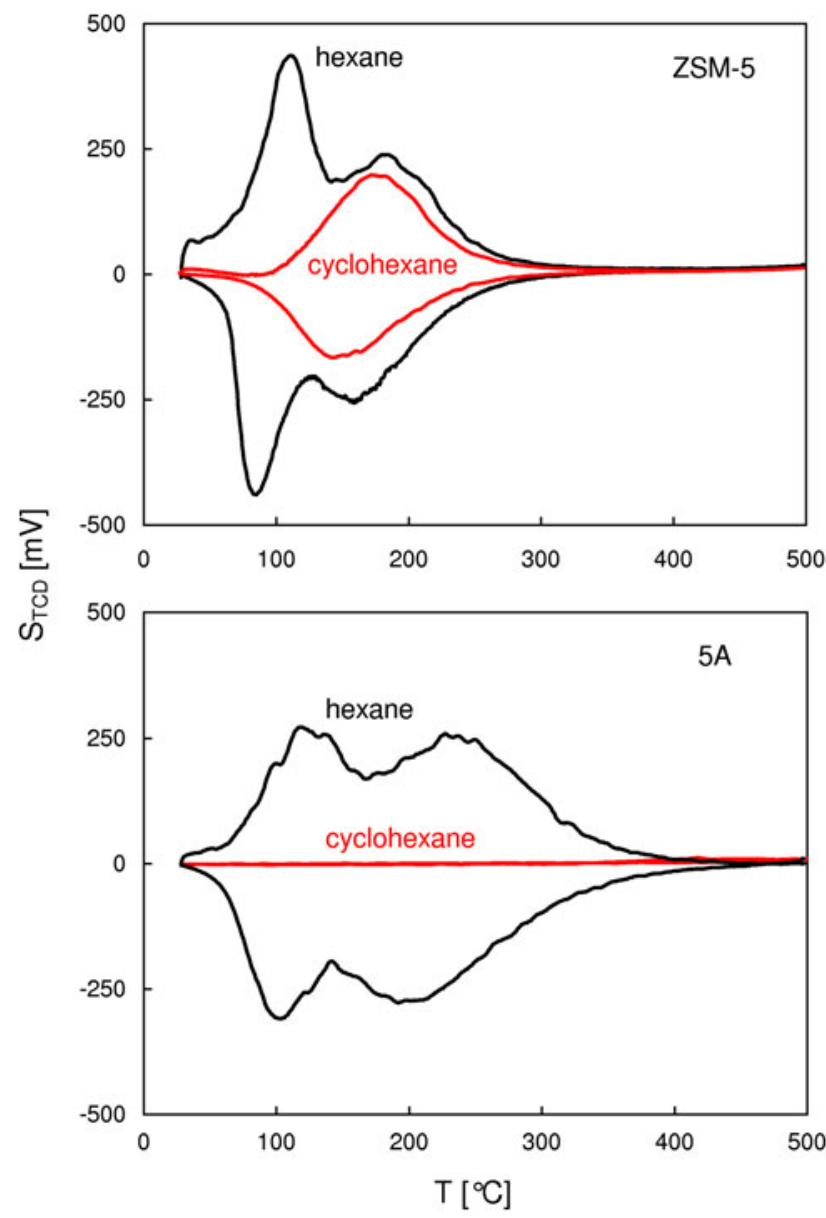

Fig. 1 QE-TPDA profiles of hexane and cyclohexane on zeolites ZSM-5 and $5 \mathrm{~A}$, measured at $10{ }^{\circ} \mathrm{C} / \mathrm{min}$. Partial pressures of hexane and cyclohexane in the carrier gas equal to 6 and 7 mbar, respectively $\frac{d \theta}{d T}=\frac{\mathrm{p} \Delta H_{a d s} \exp \left(-\frac{\Delta G_{a d s}}{R T}\right)}{\left[1+\mathrm{p} \exp \left(\frac{-\Delta G_{a d s}}{R T}\right)\right]^{2} R T^{2}}$

$\Delta G_{a d s}=\Delta H_{a d s}-T \Delta S_{a d s}$,
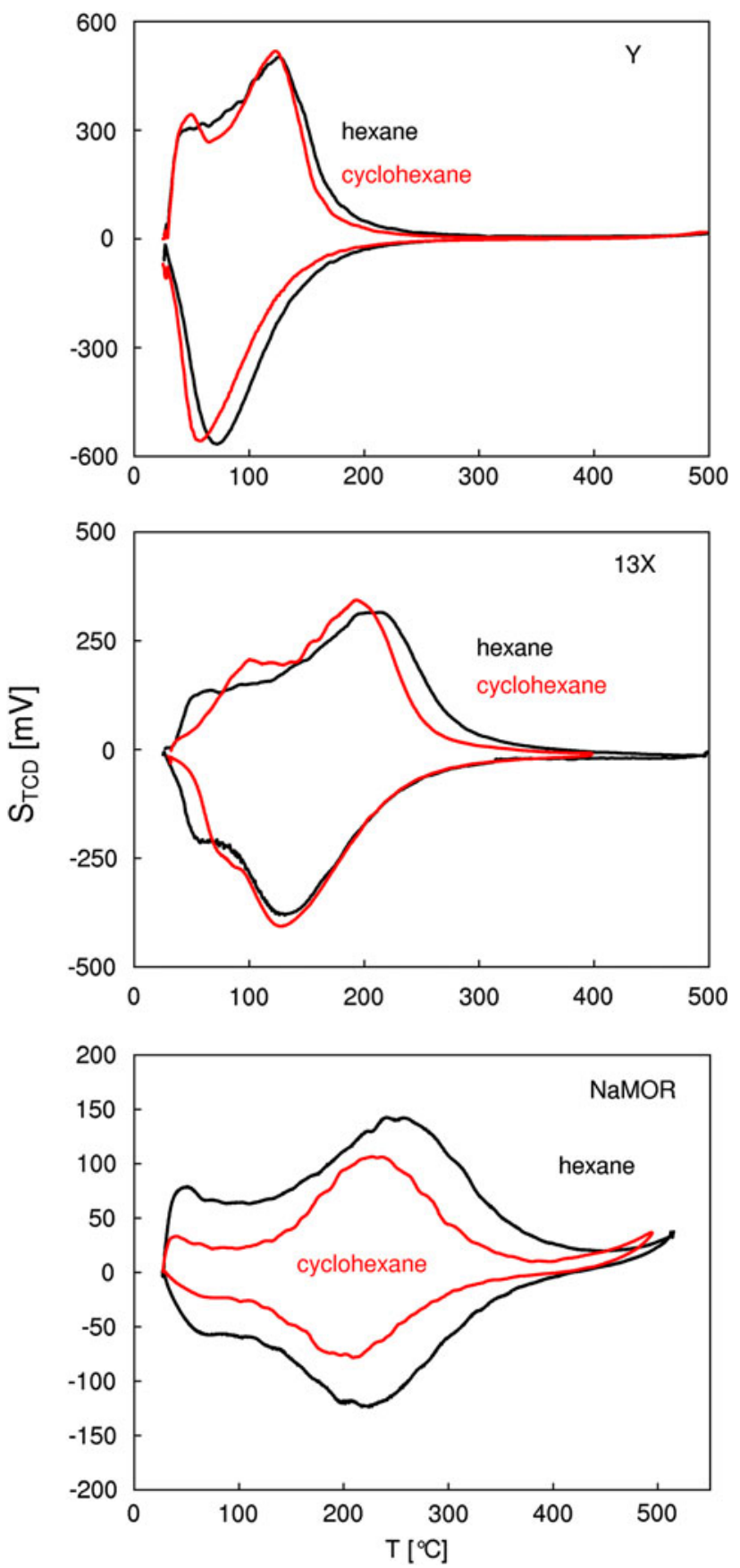

Fig. 2 QE-TPDA profiles of hexane and cyclohexane on zeolites $13 \mathrm{X}, \mathrm{Y}$ and NaMOR, measured at $10{ }^{\circ} \mathrm{C} / \mathrm{min}$. For $13 \mathrm{X}$ and $\mathrm{Y}$ partial pressures of hexane and cyclohexane equal to 6 and $7 \mathrm{mbar}$, respectively. For NaMOR they were equal to 8 and 6 mbar, respectively 
where $\mathrm{T}$ is the temperature, $\mathrm{p}$ the partial pressure of the adsorptive, $\Delta \mathrm{H}_{\mathrm{ads}}$ and $\Delta \mathrm{S}_{\mathrm{ads}}$ are the adsorption enthalpy and entropy. In the case of the two step desorption profiles observed for hexane on 5A and ZSM-5 zeolites a dual site Langmuir (DSL) function i.e. a linear combination of two single Langmuir functions (Eq. 1) was used. Least square fitting was performed using the Microsoft Excel Solver procedure.

The results of the fitting are shown in Fig. 3, and the fitting parameters (i.e. values of the adsorption enthalpy and entropy) are listed in Table 1, together with values of the micropore volume, determined by integration of the QE-TPDA profiles. Very good agreement between the experimental data and the fitted functions in almost the whole temperature range was obtained for hexane and cyclohexane on ZSM-5, and also for hexane on 5A (not shown here). For all the other adsorbate/adsorbent systems good fit could be obtained only in the high temperature range.

Values of the adsorption enthalpy and entropy of hexane calculated as the fitting parameters are in agreement with our earlier findings and the literature data (Makowski and Majda 2005, and the references therein). Differences of the corresponding parameters found for a given zeolite reflect differences in interactions of hexane and cyclohexane molecules during adsorption.

In the case of zeolites $13 \mathrm{X}$ and $\mathrm{Y}$ values of the adsorption enthalpy and entropy of cyclohexane are quite close to those found for hexane, thus indicating that interactions of both types of molecules with the walls of the supercages in the FAU framework and extraframework $\mathrm{Na}^{+}$cations do not differ considerably. Slightly larger value of the adsorption entropy loss $\left(-\Delta \mathrm{S}_{\mathrm{ads}}\right)$ obtained for cyclohexane on NaMOR may indicate that these molecules adsorbed in the 12MR channels of this zeolite have less rotational or translational freedom than the adsorbed hexane molecules. On the other hand, lower micropore volume calculated from the adsorption capacity of cyclohexane for this zeolite suggests that a part of the void space in the MOR framework (most probably 8MR side pockets perpendicular to the main $12 \mathrm{MR}$ channels) are not accessible for cyclohexane molecules, in contrary to those of hexane.

The parameters found for the two step desorption profiles of hexane observed for 5A and ZSM-5 zeolites are consistent with the "commensurate freezing" concept. Large values of the entropy loss $\left(-\Delta \mathrm{S}_{\mathrm{ads}}\right)$ found for the low temperature peaks corroborate a considerable ordering of the adsorbed molecules and suppressing of their mobility at high adsorption degrees. The lack of the low temperature desorption step in the QE-TPDA profile of cyclohexane on ZSM-5 indicates that such an ordering of these molecules does not occur. However, it should be noticed that values
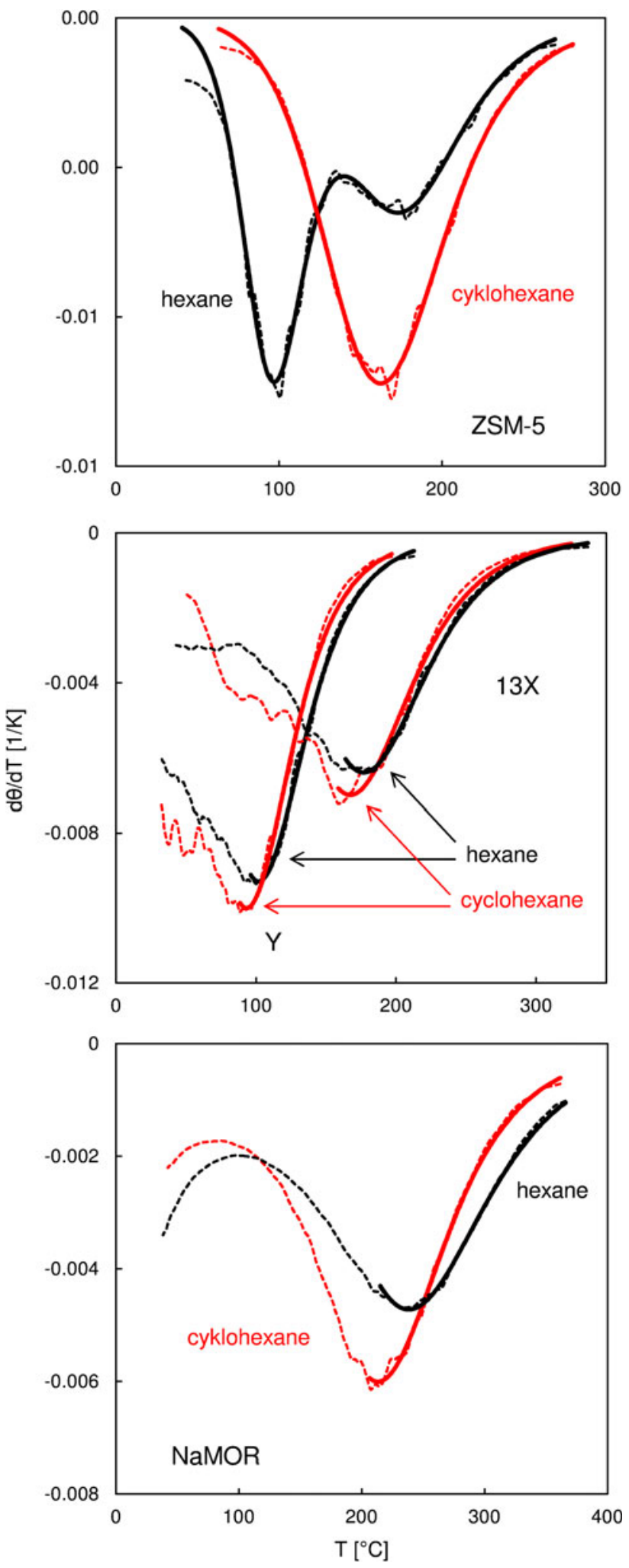

Fig. 3 Fitting of the Langmuir and the DSL functions (solid lines) to the temperature derivatives of the normalized isobars determined by integration of the QE-TPDA profiles of hexane and cyclohexane (dashed lines) 
Table 1 Values of the parameters determined for zeolites from QE-TPDA profiles of hexane and cyclohexane

\begin{tabular}{lllll}
\hline Zeolite & Sorbate & $\begin{array}{l}-\Delta \mathrm{H}_{\text {ads }} \\
(\mathrm{kJ} / \mathrm{mol})\end{array}$ & $\begin{array}{l}-\Delta \mathrm{S}_{\text {ads }} \\
(\mathrm{J} /(\mathrm{mol} \cdot \mathrm{K}))\end{array}$ & $\begin{array}{l}\mathrm{V}_{\text {micro }} \\
\left(\mathrm{cm}^{3} / \mathrm{g}\right)\end{array}$ \\
\hline 5A & Hexane $^{\text {ht }}$ & 56 & 69 & $0.136^{\mathrm{t}}$ \\
& Hexane $^{\mathrm{lt}}$ & 71 & 139 & \\
ZSM-5 & Hexane $^{\mathrm{ht}}$ & 71 & 115 & $0.129^{\mathrm{t}}$ \\
& Hexane & 90 & 199 & \\
& Cyclohexane & 63 & 102 & 0.059 \\
$\mathrm{X}$ & Hexane & 61 & 91 & 0.155 \\
& Cyclohexane & 60 & 92 & 0.155 \\
$\mathrm{Y}$ & Hexane & 57 & 106 & 0.160 \\
& Cyclohexane & 56 & 110 & 0.160 \\
\multirow{2}{*}{ NaMOR } & Hexane & 56 & 67 & 0.099 \\
& Cyclohexane & 58 & 76 & 0.053 \\
\hline
\end{tabular}

$h t$ high temperature, $l$ low temperature, $t$ total

of the adsorption enthalpy end entropy loss $\left(-\Delta \mathrm{H}_{\mathrm{ads}}\right.$ and $-\Delta \mathrm{S}_{\mathrm{ads}}$ ) for the high temperature desorption peak observed for ZSM-5 are smaller in the case of cyclohexane. This may mean that the adsorbed cyclohexane molecules are located in the intersections of 10MR channels of MFI framework where they have more rotational freedom than hexane molecules adsorbed within the channels.

\subsection{Mesoporous silicas}

The QE-TPDA profiles of both hydrocarbons measured for all the studied mesoporous silicas are plotted in Fig. 4. The partial pressures of hexane and cyclohexane during the experiments were about 24 and 15 mbar, respectively. However, as their saturation values at $25{ }^{\circ} \mathrm{C}$ are much higher (200 mbar for hexane and 130 mbar for cyclohexane), adsorption was limited only to the silica surface and capillary condensation in the mesopores did not occur. The profiles consist of one low temperature desorption maximum and adsorption minimum typical for the multilayer adsorption on the silica surface (Makowski et al. 2009). The differences in intensity for the thermodesorption profiles of hexane and cyclohexane result from the lower value of the cyclohexane partial pressure applied. But these differences were not always the same-it is worth noting that both QE-TPDA profiles obtained for SBA-15 are similar in the shape and intensity. Additionally, these profiles are broader than those obtained for any other mesoporous material. These facts might reflect differences in the morphology or roughness of the SBA-15 silica surface in comparison with other silicas.

The observed minima related to the adsorption of hydrocarbons have lower intensity of the signal in comparison to the maxima. This effect is caused by noneffective cooling down the sample in the low temperature range, shown in the inset in the Fig. 4. In the range of $25-80{ }^{\circ} \mathrm{C}$ the temperature decrease is slower than $10^{\circ} \mathrm{C} / \mathrm{min}$, which results in a slower uptake of the adsorptive from the carrier gas, and consequently in a decreased intensity of the adsorption minimum. However, this limitation does not affect the quasi equilibrium control of the thermodesorption measurements.

The QE-TPDA profiles of hexane and cyclohexane found for the studied mesoporous materials were converted into the adsorption isobars, according a similar procedure to that used for transformation of the profiles obtained for zeolites. The only difference was that the integrated profiles were not normalized. The resulting isobars (Fig. 5) were fitted with the BET function:

$V_{a d s}=\frac{V_{m}\left(\frac{p}{p_{e}}\right) C}{\left(1-\frac{p}{p_{e}}\right)\left(1+(C-1)\left(\frac{p}{p_{e}}\right)\right)}$

where $\mathrm{V}_{\text {ads }}$ denotes the specific adsorbed volume, $\mathrm{V}_{\mathrm{m}}$ - the specific volume related to the monolayer, $\mathrm{p}$-partial pressure of the adsorptive in the carrier gas, $\mathrm{p}_{\mathrm{e}}$ saturation partial pressure. Temperature dependence of $p_{e}$ and the corresponding parameters were taken from the CHERIC KDB database ${ }^{1}$ :

$\operatorname{In}\left(p_{e}\right)=A \ln (T)+\frac{B}{T}+C+D T^{2}$

Parameter C in Eq. 3 is a function of temperature depending on the difference of the condensation and adsorption enthalpy:

$C=\exp \left(\frac{\Delta H_{\text {cond }}-\Delta H_{a d s}}{R T}\right)$.

Values of the condensation enthalpy of hexane and cyclohexane were calculated from temperature dependencies of their saturation partial pressure as -30.1 and $-31.5 \mathrm{~kJ} / \mathrm{mol}$, respectively. Values of $\mathrm{V}_{\mathrm{m}}$ and $\Delta \mathrm{H}_{\mathrm{ads}}$ were calculated as the fitting parameters. Values of the specific surface area $\left(S_{\mathrm{BET}}\right)$ were obtained from $\mathrm{V}_{\mathrm{m}}$, assuming cross sectional area of hexane and cyclohexane molecules equal to 0.36 and $0.45 \mathrm{~nm}^{2}$, respectively (McClellan and Harnsberger 1967).

The BET functions, fitted in whole range of temperature, show very good agreement with the experimental data for almost all mesoporous silicas, especially for MCM-41 and HMS. However, remarkable differences between the fitted BET lines and experiment data may be noticed for the SBA-15.

The adsorption isobars derived from QE-TPDA profiles of hexane and cyclohexane were also used in determination

\footnotetext{
${ }^{1}$ Chemical Engineering Research Information Center, Korean Thermophysical Properties Data Bank (http://www.cheric.org/research/ $\mathrm{kdb} /)$.
} 

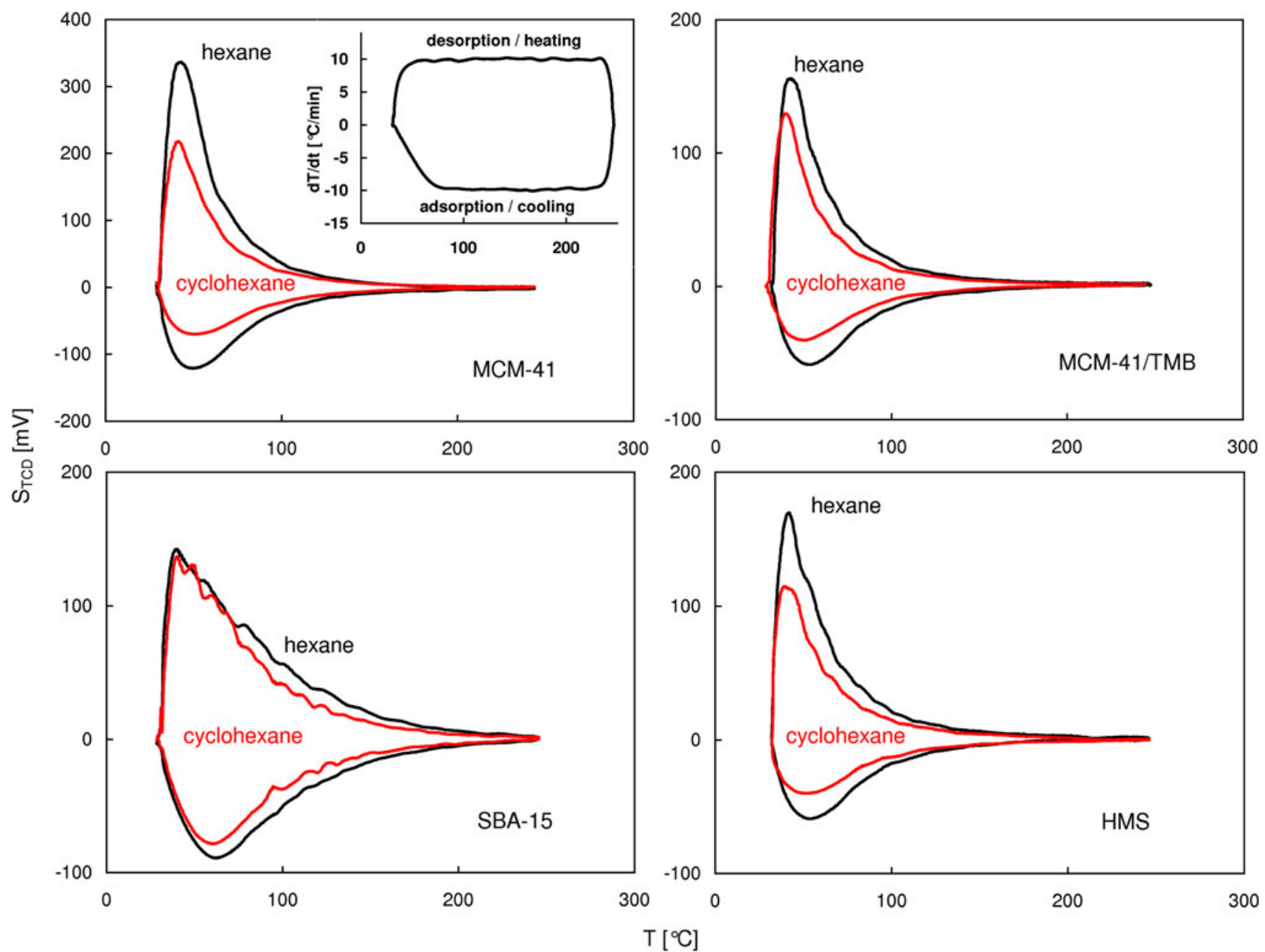

Fig. 4 QE-TPDA profiles of hexane and cyclohexane on the MCM41, MCM-41/TMB, SBA-15 and HMS mesoporous materials, measured at $10{ }^{\circ} \mathrm{C} / \mathrm{min}$. Partial pressures (in mbar) of hexane: 24

(MCM-41, SBA-15, HMS), 22 (MCM-41/TMB); of cyclohexane: 15 (MCM-41, SBA-15, HMS), 16 (MCM-41/TMB)

of the initial heats of adsorption. The Henry's law was fitted to the high-temperature sections of the isobars (corresponding to the initial $2.5 \%$ of total adsorption), after their transformation into $\ln \left(\mathrm{V}_{\text {ads }}\right)$ versus $1 / \mathrm{T}$ relations (Fig. 6).

The transformed isobars of both hydrocarbons found for MCM-41, MCM-41/TMB and HMS show practically linear dependence indicating high homogeneity of the adsorption sites on their surfaces. On the other hand, for SBA-15 the transformed isobars are curved and deviate from the Henry law in the whole temperature range.

Values of the adsorption heats and surface areas calculated by fitting the model functions are compared in Table 2. Those found for SBA-15 confirm high heterogeneity of its surface. The initial adsorption heats are close to those found for zeolites $\mathrm{Y}$ and NaMOR. This suggests that the strongest adsorption sites on the surface of SBA-15 are similar to the micropores in zeolites formed by $12 \mathrm{MR}$ windows. The largest differences between values of the Henry and BET adsorption heats noticed for this material indicate that the strongest adsorption sites represent only a small fraction of the whole surface.

Values of the adsorption heats obtained for other silicas are smaller and agree well with the literature data (Trens et al. 2004). Smaller differences between the Henry and BET adsorption heats confirm homogeneity of the surface adsorption sites in these materials, which increases with the increasing diameter of the mesopores.

Values of the BET specific surface area determined from the adsorption isobars of hexane and cyclohexane are smaller than those obtained from $\mathrm{N}_{2}$ adsorption isotherms, and there is no evident correlation between these sets of parameters. These differences may result from the fact that the fitted isobars correspond to the range of relative partial pressures of $0-0.12$, while in BET analysis of $\mathrm{N}_{2}$ adsorption isotherms was performed in the range of 0.05-0.25. However, as yet these discrepancies in the BET surface areas are not properly explained, therefore further studies are necessary to elucidate this problem. 


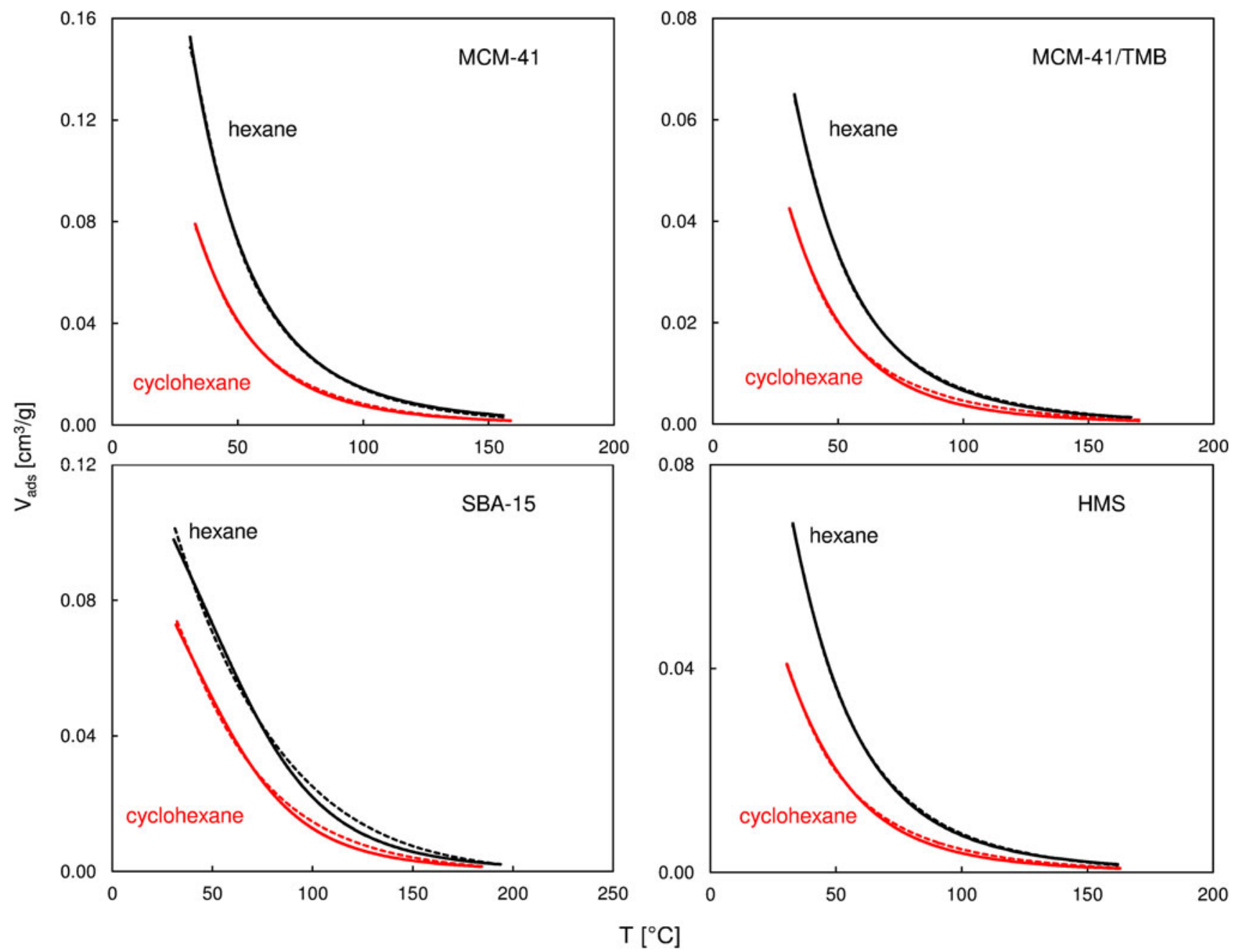

Fig. 5 Adsorption isobars of hexane and cyclohexane determined by integration of the QE-TPDA profiles (dashed lines) fitted with the BET function (solid lines)

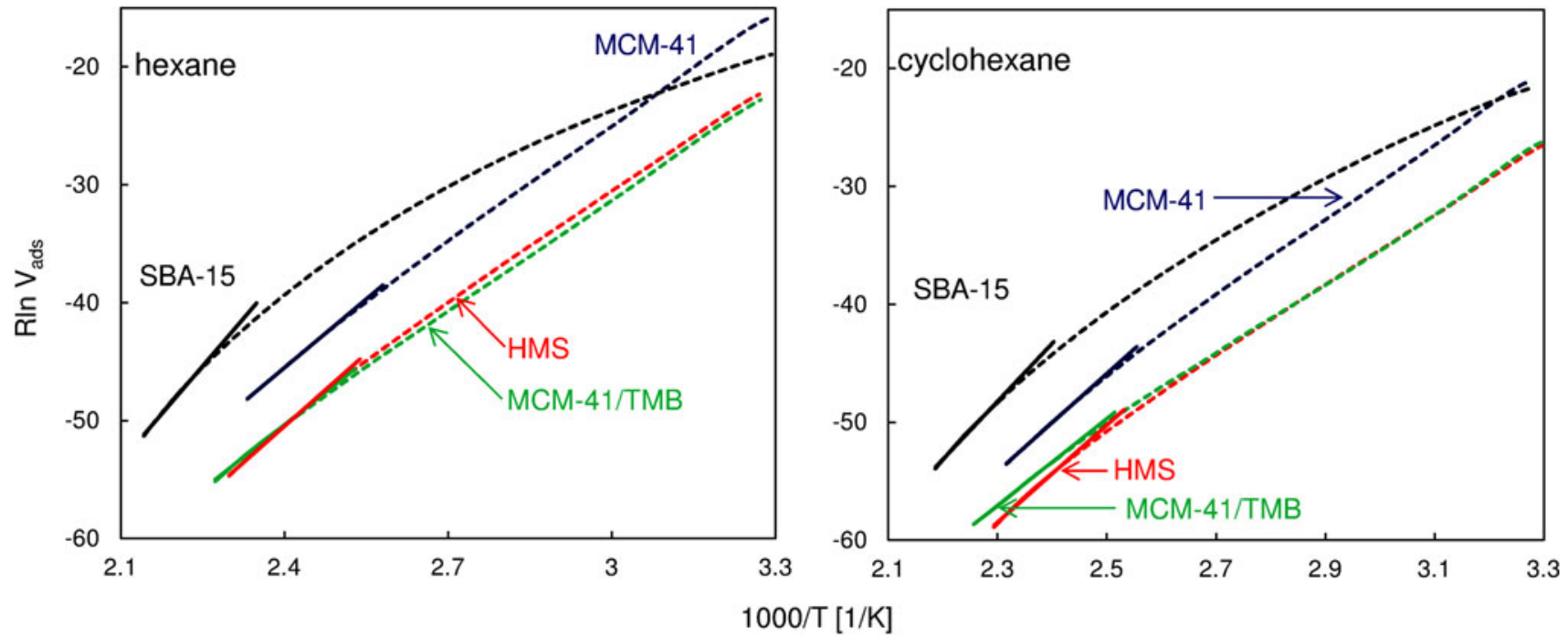

Fig. 6 The linearized adsorption isobars of hexane and cyclohexane on mesoporous silicas (dashed lines) fitted with the Henry function in the high temperatures sections (solid lines) 
Table 2 Values of the parameters determined for mesoporous materials from the hexane and cyclohexane adsorption isobars and nitrogen adsorption isotherms

\begin{tabular}{|c|c|c|c|c|c|c|c|c|}
\hline \multirow[t]{3}{*}{ Material } & \multicolumn{5}{|c|}{$-\Delta \mathrm{H}_{\mathrm{ads}}(\mathrm{kJ} / \mathrm{mol})$} & \multicolumn{3}{|c|}{$\mathrm{S}_{\mathrm{BET}}\left(\mathrm{m}^{2} / \mathrm{g}\right)$} \\
\hline & \multirow{2}{*}{$\begin{array}{l}\mathrm{N}_{2} \\
\text { BET }\end{array}$} & \multicolumn{2}{|c|}{ Hexane } & \multicolumn{2}{|c|}{ Cyclohexane } & \multirow[t]{2}{*}{$\mathrm{N}_{2}$} & \multirow[t]{2}{*}{ Hexane } & \multirow[t]{2}{*}{ Cyclohexane } \\
\hline & & Henry & BET & Henry & BET & & & \\
\hline MCM-41 & 8.6 & 38 & 33 & 41 & 35 & 879 & 867 & 580 \\
\hline MCM-41/TMB & 8.5 & 36 & 34 & 36 & 35 & 612 & 343 & 270 \\
\hline SBA-15 & 9.1 & 54 & 40 & 48 & 40 & 720 & 172 & 211 \\
\hline HMS & 8.4 & 41 & 34 & 38 & 36 & 954 & 311 & 215 \\
\hline
\end{tabular}

\section{Conclusions}

The presented results show that comparison of the QE-TPDA profiles of hexane and cyclohexane is suitable for discrimination of zeolites containing pores formed by 8 , 10 and 12 MR windows. It has been demonstrated that cyclohexane is not adsorbed within the LTA type framework, while for the FAU type zeolites its thermodesorption profiles are very similar to those of hexane.

It has been showed that application of both hydrocarbons in characterization of mesoporous silicas gave similar results, confirming high heterogeneity of the SBA-15 silica surface, in contrast to more homogeneous MCM-41, MCM-41/TMB and HMS. It has also been found that analysis of the adsorption isobars determined from the QE-TPDA profiles gives reasonable values of the adsorption heat, while the corresponding BET surface areas differ considerably from those calculated from $\mathrm{N}_{2}$ adsorption isotherms.

Acknowledgments This work was in part supported by the International $\mathrm{PhD}$-studies programme at the Faculty of Chemistry Jagiellonian University within the Foundation for Polish Science MPD Programme co-financed by the EU European Regional Development Fund.

Open Access This article is distributed under the terms of the Creative Commons Attribution License which permits any use, distribution, and reproduction in any medium, provided the original author(s) and the source are credited.

\section{References}

Eder, F., Lercher, J.A.: Alkane sorption in molecular sieves: the contribution of ordering, intermolecular interactions, and sorption on Brønsted acid sites. Zeolites 18, 75-81 (1997)

Majda, D., Makowski, W., Mańko, M.: Pore size distribution of micelle-templated silicas studied by thermoporosimetry using water and n-heptane. J. Therm. Anal. Calorim. 109, 663-669 (2012)

Makowski, W., Majda, D.: Equilibrated thermodesorption studies of adsorption of $n$-hexane and n-heptane on zeolites Y, ZSM-5 and ZSM-11. Appl. Surf. Sci. 252, 707-715 (2005)
Makowski, W.: Quasi-equilibrated temperature programmed desorption and adsorption: a new method for determination of the isosteric adsorption heat. Thermochim. Acta 454, 26-32 (2007)

Makowski, W., Ogorzałek, Ł.: Determination of the adsorption heat of n-hexane and n-heptane on zeolites beta, L, 5A, 13X, Y and ZSM- 5 by means of quasi-equilibrated temperature-programmed desorption and adsorption (QE-TPDA). Thermochim. Acta 465, 30-39 (2007)

Makowski, W., Chmielarz, L., Kuśtrowski, P.: Determination of the pore size distribution of mesoporous silicas by means of quasiequilibrated thermodesorption of n-nonane. Microporous Mesoporous Mater. 120, 257-262 (2009)

Makowski, W., Leżańska, M., Mańko, M., Włoch, J.: Porosity and surface properties of mesoporous silicas and their carbon replicas investigated with quasi-equlibrated thermodesorption of $n$-hexane and $n$-nonane. J. Porous Mater. 17, 737-745 (2010)

Mańko, M., Gil, B., Janus, R., Kuśtrowski, P., Makowski, W.: Characterization of the porosity and surface chemistry of mesoporous silicas by quasi-equilibrated thermodesorption of 1-butanol and $n$-nonane. Thermochim. Acta 511, 82-88 (2010)

McClellan, A.L., Harnsberger, H.F.: Cross-sectional areas of molecules adsorbed on solid surfaces. J. Colloid Interface Sci. 23, 577-599 (1967)

Otake, M.: Shape selectivity investigation based on sorption data. J. Catal. 142, 303-311 (1993)

Pańczyk, T., Gac, W., Pańczyk, M., Borowiecki, T., Rudziński, W.: On the equilibrium nature of thermodesorption processes. TPD$\mathrm{NH} 3$ studies of surface acidity of $\mathrm{Ni} / \mathrm{MgO}-\mathrm{Al} 2 \mathrm{O} 3$ catalysts. Langmuir 22, 6613-6621 (2006)

Roque-Malherbe, R., Diaz-Castro, F.: Calculation of the energy of adsorption of n-paraffins in nanoporous crystalline and ordered acid catalysts, and its relationship with the activation energy of the monomolecular catalytic cracking reaction. J. Mol. Catal. A Chem. 280, 194-202 (2008)

Ruthven, D.M.: Characterization of zeolites by sorption capacity measurements. In: Robson, H. (ed.) Verified Syntheses of Zeolitic Materials, pp. 61-66. Elsevier, Amsterdam (2001)

Sivasankar, N., Vasudevan, S.: Adsorption of $n$-hexane in Zeolite5A: a temperature-programmed desorption and ir-spectroscopic study. J. Phys. Chem. B 109, 15417-15421 (2005)

Smit, B., Maesen, T.L.M.: Commensurate 'freezing' of alkanes in the channels of a zeolite. Nature 374, 42-44 (1995)

Thommes, M.: Physical adsorption characterization of nanoporous materials. Chem. Eng. Techol. 82, 1059-1073 (2010)

Traa, Y., Sealy, S., Weitkamp, J.: Characterization of the pore size of molecular sieves using molecular probes. Mol. Sieves 5, 103-154 (2007)

Trens, P., Tanchoux, N., Maldonado, D., Galarneau, A., Di Renzo, F., Fajula, F.: Study of $n$-hexane adsorption in MCM-41 mesoporous materials: a scaling effect approach of capillary condensation processes. New J. Chem. 28, 874-879 (2004) 